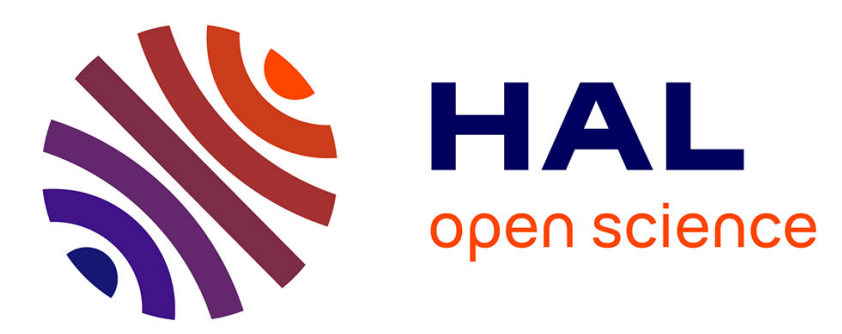

\title{
Testing for Unit Root against LSTAR Model: Wavelet Improvement under GARCH Distortion
}

Ghazi Shukur

\section{To cite this version:}

Ghazi Shukur. Testing for Unit Root against LSTAR Model: Wavelet Improvement under GARCH Distortion. Communications in Statistics - Simulation and Computation, 2009, 39 (02), pp.277-286. 10.1080/03610910903443964 . hal-00550609

\section{HAL Id: hal-00550609 https://hal.science/hal-00550609}

Submitted on 29 Dec 2010

HAL is a multi-disciplinary open access archive for the deposit and dissemination of scientific research documents, whether they are published or not. The documents may come from teaching and research institutions in France or abroad, or from public or private research centers.
L'archive ouverte pluridisciplinaire HAL, est destinée au dépôt et à la diffusion de documents scientifiques de niveau recherche, publiés ou non, émanant des établissements d'enseignement et de recherche français ou étrangers, des laboratoires publics ou privés. 


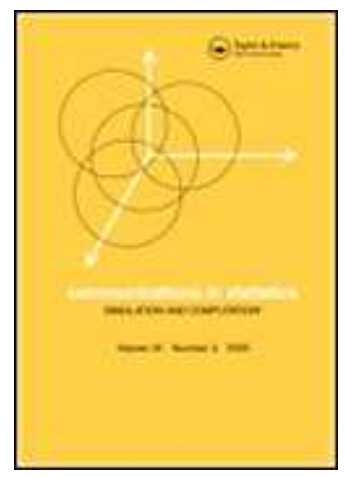

\section{Testing for Unit Root against LSTAR Model: Wavelet Improvement under GARCH Distortion}

\begin{tabular}{|r|l|}
\hline Journal: & Communications in Statistics - Simulation and Computation \\
\hline Manuscript ID: & LSSP-2009-0024.R1 \\
\hline Manuscript Type: & Original Paper \\
\hline Author: & $20-$ Oct-2009 \\
\hline Complete List of Authors: & $\begin{array}{l}\text { shukur, ghazi; Economics and Statistics, School of Management and } \\
\text { Economics; Jönköping (Jonkoping) International Businesss School, } \\
\text { Economics and Statistics }\end{array}$ \\
\hline Keywords: & $\begin{array}{l}\text { Unit root Test, Dickey-Fuller test, STAR model, GARCH (1, 1), } \\
\text { Wavelet method, MODWT }\end{array}$ \\
\hline Abstract: & $\begin{array}{l}\text { In this paper, we propose a Nonlinear Dickey-Fuller (NDF) test for } \\
\text { unit root against LSTAR (1) model with time as the transition } \\
\text { variable. The asymptotic distribution of the test is analytically } \\
\text { derived while the small sample distributions are investigated by } \\
\text { Monte Carlo experiment. The results have shown that there is a } \\
\text { serious size distortion for the NDF test under GARCH errors which } \\
\text { lead to an over-rejection of the unit root null hypothesis. Wavelet } \\
\text { technique has been used to improve the size property and an } \\
\text { empirical example is used to compare our test with the traditional } \\
\text { Dickey-Fuller test. }\end{array}$ \\
\hline & \\
\hline
\end{tabular}

\section{S) ScholaroNE" \\ Manuscript Central}




\begin{abstract}
In this paper, we propose a Nonlinear Dickey-Fuller $F$ test for unit root against first order Logistic Smooth Transition Autoregressive LSTAR (1) model with time as the transition variable. The Nonlinear Dickey-Fuller $F$ test statistic is established under the null hypothesis of random walk without drift and the alternative model is a nonlinear LSTAR (1) model. The asymptotic distribution of the test is analytically derived while the small sample distributions are investigated by Monte Carlo experiment. The size and power properties of the test have been investigated using Monte Carlo experiment. The results have shown that there is a serious size distortion for the test when GARCH errors appear in the Data Generating Process (DGP), which lead to an over-rejection of the unit root null hypothesis. To solve this problem, we use the Wavelet technique to count off the GARCH distortion and to improve the size property of the test under GARCH error. We also discuss the asymptotic distributions of the test statistics in GARCH and wavelet environments.
\end{abstract}

Keywords: Unit root Test, Dickey-Fuller $F$ test, STAR model, GARCH $(1,1)$, Wavelet method, MODWT

\title{
I. Introduction
}

Empirical studies show that many economic variables display nonlinear features where the economic behaviors change when certain variables lie in different regions. To capture such features, several nonlinear models have been introduced. Among them, STAR models allow nonlinear structures between the data regimes to be described with a smooth regime transition function. They are of particular interest in 
macroeconomics which always contains mass of economic agents, where even if the decisions are made discretely, the aggregated behaviors will show smooth regime changes (see Teräsvirta, 1994). However, before applying nonlinear models such as STAR models, testing linearity against nonlinearity is essential, and unit root tests against nonlinear model need further cautious consideration. In STAR models, Eklund (2003) proposed unit root tests against LSTAR with transition variables being the lagged dependent variables. Later, He and Sandberg (2006) proposed the nonlinear Dickey-Fuller $\rho$ and $t$ test statistics with time as the transition variable. In this paper we will first derive Nonlinear Dickey-Fuller $F$ test of unit root against LSTAR models with time as the transition variable.

We next investigate the size property of the Nonlinear Dickey-Fuller $F$ test when the error in the DGP shows conditional heteroskedasticity as a $\operatorname{GARCH}(1,1)$ model. As ARCH/GARCH models are always employed to model the conditional variance without paying enough attention to the specification of the conditional mean, and that the misspecification may lead to further inconsistent estimates, specification tests including unit root test under ARCH/GARCH error has attracted much attention. Peter and Veloce (1988), Kim and Schmidt (1993), Cook (2006) showed that Dickey-Fuller test is generally not robust in the near integrated situation in GARCH error, especially when the GARCH process exhibits a high degree of volatility. Therefore, to improve the test property, numerous studies pay attention to deriving unit root test based on Maximum Likelihood Estimation (MLE), which jointly estimates the parameters of unit root model and the GARCH error model. (see Seo,1999; Ling and Li, 1998). However, the MLE is not a perfect solution to the GARCH error problem. Charles and Darné (2008) re-examined Seo's test which is based on MLE and showed that the empirical size and power of the Dickey-Fuller test is generally better than Seo's test when the GARCH paramter $\beta$ is superior to $\mathrm{ARCH}$ parameter $\alpha$ (The definition of $\beta$ and $\alpha$ please refer to Section IV). Moreover, in our LSTAR model, if we use MLE method, the estimated dimensional parameter space is larger than the linear case and it 
will be numerically quite complicated to obtain. Thus in this paper, we consider an alternative to MLE method to improve the unit root test under GARCH error.

We apply the wavelet method, which has been widely used after its theoretic foundation in 1980s (see Grossmann and Morelet, 1984 and Mallat, 1989). In economics, Schleicher (2002) mentioned that since economic behaviors take place at different frequencies, the wavelet method can catch landscape characteristics in addition to the microscopic detail in economic areas. In this paper, we use the wavelet method to count off the finest local behavior of the series in the form of conditional heteroskedasticity in GARCH errors, whose information is caught by the highest scale in wavelet coefficients. The same logic can be found in Schleicher (2002), who pointed out that lower scales hold most of the energy of the unit root process and that non-lasting disturbances are captured by the higher scale coefficients. This logic is also reflected in Fan and Gençay (2006), who stated that the spectrum of a unit root process is infinite at frequency 0 . They proposed a unit root test on the perspective of the frequency domain as the test is the ratio of the energy of the low frequency scale to the total energy of the time series. Here our Nonlinear Dickey-Fuller $F$ test statistic is in a time domain where we use the scaling coefficient directly in the test statistics; in this way, the asymptotic distribution of the test statistics will not be influenced under the wavelet environment. We use Maximal Overlap Discrete Wavelet Transform (MODWT) as it has no restriction on the sample size and LA (8) wavelet filter as it has better band pass character. For more information about the MODWT methods and LA filter, we refer to Vidakovic (1998), Percival and Walden (2000), and to Gençay Selcuk and Whicher (2001b).

The paper is organized as follows. Section II presents the LSTAR model, the procedure for testing unit root against the LSTAR alternatives, the asymptotic properties and the finite sample distribution of the test statistics. Section III investigates the size and power property of the test, and offers an empirical example. Section IV shows the size distortion of the test statistics under GARCH $(1,1)$ error. 
Section V presents the wavelet size improvement of the small samples and the asymptotical distribution. Concluding remarks can be found in the final section. All proofs of theorems in this paper are omitted to save place, but available from the authors up on request.

\section{Model, Test procedure, The Nonlinear Dickey-Fuller $F$ test}

In STAR models, the LSTAR model can catch the asymmetric feature of a process in two extreme states: when the economic contractions are always more violent, and when expansions are more stationary and persistent. This paper only considers the nonlinear LSTAR models with the following structure:

$$
\begin{aligned}
& y_{t}=\pi_{10}+\pi_{11} y_{t-1}+\left(\pi_{20}+\pi_{21} y_{t-1}\right) F(t, \gamma, c)+u_{t} \\
& F(t ; \gamma, c)=\frac{1}{(1+\exp \{-\gamma(t-c)\})}-\frac{1}{2} .
\end{aligned}
$$

Here the transition variable is defined as time $t$, which implies that the equilibrium regimes switch as the time evolves. Parameter $\gamma$ determines the speed of transition from one extreme regime to another at time $c$ : the larger the $\gamma$ is, the steeper the transition function will be. Meanwhile, set $c$ fixed, as $\gamma \rightarrow \infty$, the function turns into a step function of $t$ and the model becomes a two regimes threshold autoregressive model (TAR). When setting $t$ and $c$ fixed, the situation when $\gamma \rightarrow 0$ leads the resulting model to be linear. Therefore, the linear hypothesis is equivalent to the hypothesis: $\gamma=0$. Our goal is to test the null hypothesis of a random walk without drift against the nonlinear LSTAR (1) model. The null hypothesis can be expressed as the following parameter restrictions:

$H_{0}: \gamma=0, \pi_{10}=0, \pi_{11}=1$.

Since $\gamma=0$ will lead to an identification problem under the null hypotheses (see Teräsvirta, 1994), we follow the approach used by Luukkonen, Saikkonen and 
Teräsvirta (1988), by applying a Taylor expansion of the $F(t, \gamma, c)$ with $\gamma$ around 0 . As the first-order expansion will lead to low power if the transition takes place only in the drift, we show also the third-order Taylor expansion which is more robust:

$$
\begin{aligned}
& F_{1}(t ; \gamma, c)=\frac{\gamma(t-c)}{4}+o(\gamma) . \\
& F_{3}(t ; \gamma, c)=\frac{\gamma(t-c)}{4}+\frac{\gamma^{3}(t-c)^{3}}{48}+o\left(\gamma^{3}\right) .
\end{aligned}
$$

Substituting equations (2) and (3) into equations (1), we obtain the following auxiliary regressions:

Order $1: y_{t}=s_{1 t}^{\prime} \lambda_{1}+\left(y_{t-1} s_{1 t}\right)^{\prime} \varphi_{1}+u_{1 t}$.

Order $3: y_{t}=s_{3 t}^{\prime} \lambda_{3}+\left(y_{t-1} s_{3 t}\right)^{\prime} \varphi_{3}+u_{3 t}$.

$$
s_{1 t}=\left(\begin{array}{l}
1 \\
t
\end{array}\right), \lambda_{1}=\left(\begin{array}{l}
\lambda_{10} \\
\lambda_{11}
\end{array}\right), \varphi_{1}=\left(\begin{array}{l}
\varphi_{10} \\
\varphi_{11}
\end{array}\right) ; s_{3 t}=\left(\begin{array}{l}
1 \\
t \\
t^{2} \\
t^{3}
\end{array}\right), \lambda_{3}=\left(\begin{array}{l}
\lambda_{30} \\
\lambda_{31} \\
\lambda_{32} \\
\lambda_{33}
\end{array}\right), \varphi_{3}=\left(\begin{array}{l}
\varphi_{30} \\
\varphi_{31} \\
\varphi_{32} \\
\varphi_{33}
\end{array}\right)
$$

The unit root test in the nonlinear time series model is a joint test of both unit root and linearity. The corresponding auxiliary null hypotheses are:

$$
H_{0 m}: \lambda_{m i}=0, \forall i ; \varphi_{m 0}=1, \varphi_{m j}=0, j \geq 1 ; m=1,3 \text {. }
$$

Following the above auxiliary regressions and null hypothesis, we now derive the unit root test statistics and investigate their distribution properties. Here we assume that the error terms in equations (1) are independent identically distributed (i.i.d.). In the distribution form, the $W($.$) represents a standard Brownian motion on [0,1]$.

Assumption 1: Let $\left\{u_{t}\right\}$ is i.i.d.with $E\left(u_{t}\right)=0, \operatorname{Var}\left(u_{t}\right)=\sigma_{u}^{2}$, and $E\left(u_{t}^{4}\right)<\infty$.

Theorem 2: Assume that the following models $y_{t}=s_{m t} \lambda_{m}+\left(y_{t-1} s_{m t}\right)^{\prime} \varphi_{m}+u_{m t}$ hold, and assume that $\left(u_{m t}\right)_{t=1}^{\infty}$ fulfills Assumption 1, then for $m=1,3$, we have:

$$
\hat{\psi}_{m}-\psi_{m} \stackrel{p}{\rightarrow} 0, \quad \Upsilon_{m}\left(\hat{\psi}_{m}-\psi_{m}\right) \stackrel{L}{\rightarrow} \Psi_{m}^{-1} \Pi_{m}, \quad s_{m}^{2} \Upsilon_{m}\left(\sum x_{m t} x_{m t}^{\prime}\right)^{-1} \Upsilon_{m} \stackrel{L}{\rightarrow} \sigma_{u}{ }^{2} \Psi_{m}^{-1}
$$

Where the parameters are defined as follows:

$$
\Upsilon_{1}=\operatorname{diag}\left\{T_{1}\right\}, T_{1}=\left[T^{1 / 2} T^{3 / 2} T T^{2}\right],
$$




$$
\begin{aligned}
& \Upsilon_{3}=\operatorname{diag}\left\{T_{3}\right\}, T_{3}=\left[T^{1 / 2} T^{3 / 2} T^{5 / 2} T^{7 / 2} T T^{2} T^{3} T^{4}\right], \\
& \hat{\psi}_{m}=\left(\begin{array}{l}
\hat{\lambda}_{m} \\
\hat{\varphi}_{m}
\end{array}\right), \psi_{m}=\left(\begin{array}{l}
\lambda_{m} \\
\varphi_{m}
\end{array}\right), x_{m t}=\left[\begin{array}{l}
s_{m t} \\
y_{t-1} s_{m t}
\end{array}\right], \Psi_{m}=\left[\begin{array}{ll}
A_{m} & \sigma_{u} B_{m} \\
\sigma_{u} B_{m}^{\prime} & \sigma_{u}^{2} C_{m}
\end{array}\right], \Pi_{m}=\left[\begin{array}{l}
\sigma_{u} D_{m} \\
\sigma_{u}^{2} E_{m}
\end{array}\right], \\
& A_{m}=\left[a_{i j}\right]_{(m+1)^{*}(m+1)}, B_{m}=\left[b_{i j}\right]_{(m+1)^{*}(m+1)}, C_{m}=\left[c_{i j}\right]_{(m+1)^{*}(m+1)}, D_{m}=\left[d_{i}\right]_{(m+1)^{*} 1}, E_{m}=\left[e_{i}\right]_{(m+1)^{* 1}}, \\
& a_{i j}=T^{-(i+j-1)} \sum_{t=1}^{T} t^{i+j-2}, \quad b_{i j}=\int_{0}^{1} r^{i+j-2} W(r) d r, \quad c_{i j}=\int_{0}^{1} r^{i+j-2} W(r)^{2} d r \\
& d_{i}=W(1)-(i-1) \int_{0}^{1} r^{i-2} W(r) d r, \quad e_{i}=\frac{\left(W(1)^{2}-(i-1) \int_{0}^{1} r^{i-2} W(r)^{2} d r-1 / i\right)}{2} .
\end{aligned}
$$

Based on Theorem 1, under the null hypothesis $H_{0}: R_{m} \psi_{m}=r_{m}$, we have the Nonlinear Dickey-Fuller $F$ test statistic as follows:

$$
\begin{aligned}
F_{m}= & \left(\hat{\psi}_{m}-\psi_{m}\right)^{\prime} R_{m}{ }^{\prime}\left\{s_{m}^{2} R_{m}\left(\sum x_{m t} x_{m t}^{\prime}\right)^{-1} R_{m}\right\}^{-1} R_{m}\left(\hat{\psi}_{m}-\psi_{m}\right) / 2 \\
= & \left(\hat{\psi}_{m}-\psi_{m}\right)^{\prime} R_{m} \Upsilon_{m} *\left\{s_{m}^{2} \Upsilon_{m} R_{m}\left(\sum x_{m t} x_{m t}^{\prime}\right)^{-1} R_{m} \Upsilon_{m}\right\}^{-1} * \Upsilon_{m} R_{m}\left(\hat{\psi}_{m}-\psi_{m}\right) / 2 \\
& \stackrel{L}{\longrightarrow}\left(\Psi_{m}^{-1} \Pi_{m}\right)^{\prime}\left\{\sigma_{u}{ }^{2} \Psi_{m}^{-1}\right\}^{-1}\left(\Psi_{m}^{-1} \Pi_{m}\right) / 2=\Pi_{m} \Psi_{m}^{-1} \Pi_{m} / 2 \sigma_{u}{ }^{2} .
\end{aligned}
$$

Where: $R_{m}=I_{2^{*}(m+1)}, r_{1}^{\prime}=\left[\begin{array}{llll}0 & 0 & 1 & 0\end{array}\right], r_{3}^{\prime}=\left[\begin{array}{llllllll}0 & 0 & 0 & 0 & 1 & 0 & 0 & 0\end{array}\right]$.

To find out the finite-sample distributions of the test, we generate data from the model $y_{t}=y_{t-1}+u_{t}$ where $u_{t} \sim$ n.i.d. $(0,1)$ with desired sample sizes. The number of Monte Carlo replication is 20000 . To avoid high parameter dimension which shows in thirdorder Taylor expansion, we only report the critical value table with Taylor expansion of Order 1, and we also only use this case in the following part of the paper.

Table 1. Critical values for the Nonlinear D-F $F$ test

\begin{tabular}{|lllllllll|}
\hline $\mathrm{T}$ & $99 \%$ & $97.5 \%$ & $95 \%$ & $90 \%$ & $10 \%$ & $5 \%$ & $2.5 \%$ & $1 \%$ \\
\hline 50 & 1.1282 & 1.3710 & 1.6378 & 2.0181 & 6.7710 & 7.7315 & 8.6741 & 9.7927 \\
\hline 100 & 1.2205 & 1.4894 & 1.7665 & 2.1512 & 7.0376 & 8.1185 & 9.2100 & 10.4860 \\
\hline 250 & 1.2500 & 1.5324 & 1.8055 & 2.1816 & 7.2652 & 8.4135 & 9.4990 & 10.7145 \\
\hline 500 & 1.2850 & 1.5682 & 1.8660 & 2.2380 & 7.2687 & 8.3699 & 9.3918 & 10.6378 \\
\hline
\end{tabular}




\section{Size and power property of the test, Empirical Example}

We again use the Monte Carlo method to investigate the size and power properties of our test statistics. We ignore the size table as it is unbiased due to that we use same Monte Carlo experiment when simulating the critical value table. Thus we only examine the power property of the test. As we are more interested in the variation of the dynamic parameters, we set the drift parameter stable with $\pi_{10}=0, \pi_{20}=1$. We also set the transition speed parameter $\gamma=1$, and the transition time $c=T / 2$. Thus the changing parameters are only dynamical parameters $\pi_{11}, \pi_{21}$. We also impose the Lagrange stability condition $\pi_{11}+\pi_{21} \in(0,1)$ with $\pi_{11}+\pi_{21}=0.9$ to ensure the stable trajectories (Tong (1990)). To observe the power changes with the nonlinear dynamics impact measured by $\pi_{21}$, we set: $\pi_{11} \in(0.1,0.3,0.4,0.5,0.6), \pi_{21} \in(0.8,0.6,0.5,0.4,0.3)$.

Table 2. Power property for the test

\begin{tabular}{|clllll|}
\hline & $\pi_{11}=0.6$ & $\pi_{11}=0.5$ & $\pi_{11}=0.4$ & $\pi_{11}=0.3$ & $\pi_{11}=0.1$ \\
$\mathrm{~T}$ & $\pi_{21}=0.3$ & $\pi_{21}=0.4$ & $\pi_{21}=0.5$ & $\pi_{21}=0.6$ & $\pi_{21}=0.8$ \\
\hline 50 & 0.1925 & 0.3869 & 0.6056 & 0.8108 & 0.9820 \\
\hline 100 & 0.7024 & 0.9329 & 0.9925 & 0.9995 & 1 \\
250 & 0.9400 & 1 & 1 & 1 & 1 \\
\hline 500 & 1 & 1 & 1 & 1 & 1 \\
\hline
\end{tabular}

Table 2 shows that for small sample 50, the power depends mainly on the proportions of the nonlinear parts; the higher the nonlinear part, the better power is. With sample size increases to 250 and 500 , the power of the test reaches 1 .

We now show an empirical example to compare our Nonlinear Dickey-Fuller $F$ test to the traditional Dickey-Fuller $F$ test. For the unemployment rates in 10 OECD counties $^{1}$ from 1955 to 1999 and by using the Dickey-Fuller $F$ test, the unit root reject none of them, while using the Nonlinear $F$ test, the unit root was rejected in 3 series: Germany, Japan, France. We use the data from France for more detailed procedure.

\footnotetext{
${ }^{1}$ Austria, Denmark, Finland, France, Germany, Japan, Netherland, Norway, Sweden, Belgium
} 


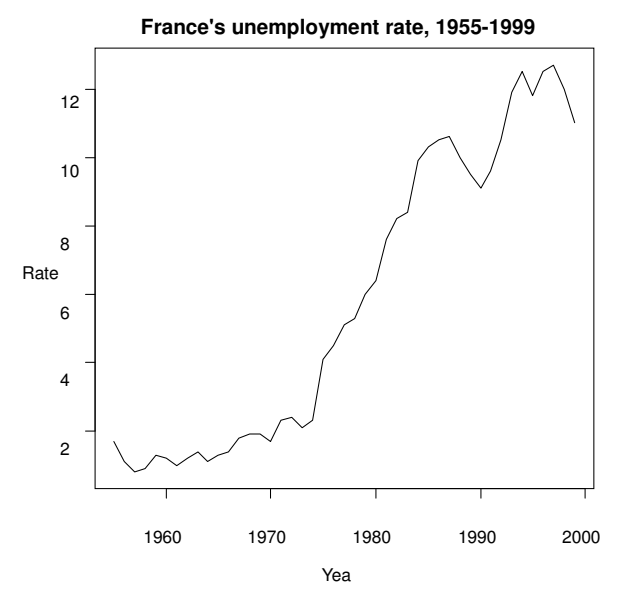

Figure 1. France's unemployment rate, 1955- 1999

In Figure 1, we can see that the time series shows an obvious data break around 1975, and the whole series is divided into two data periods with a smooth region changes. Thus we suppose that a STAR model should be a good choice. For linear DickeyFuller $F$, we first fit the data with an order one autoregressive model and the AR(1) process is: $y_{t}=0.2274+0.9971 y_{t-1}+u_{t}$ with sum of residue squares 15.04 and $F$ statistic 2.7538 , after we compare it to the critical value 4.86 in Table B.7 in Hamilton, J.D. (1994), the unit root is not rejected at the 5\% level. However, from the graph we can see that for the unemployment rate data, the series are initially at an equilibrium state but after around 1975 there shows another state, and the whole series shows a nonlinear structure. Therefore, the Dickey-Fuller $F$ test may be not valid with its linearity assumption. Chow test is used to test the series linearity against a single break at 1976 and we obtain the $F_{\text {Chow }}$ statistic: $F_{\text {Chow }}=0.2369$. At the $20 \%$ critical value, we reject the null hypothesis. Next we apply our Nonlinear test from the auxiliary model $y_{t}=-0.5518+0.0595 t+0.0834 y_{t-1}-0.06 t y_{t-1}+u_{t}$.With sum of residue squares 11.58 and $F_{N L}$ statistic 9.3687, after comparing it to 7.7315 in Table 1, the null hypothesis of a unit root is rejected at the 5\% level. Then we are building a LSTAR model by Nonlinear Least Square (NLS) regression with $t$ from 2 to 45 (correspond to the year from 1956 to 1999): 
$y_{t}=2.3243+0.3797 y_{t-1}+\left(2.5870+0.6153 y_{t-1}\right)\left[\frac{1}{(1+\exp \{-0.3408(t-20.6567)\})}-\frac{1}{2}\right]+u_{t}$

The estimation of $c$ is 20.6567 , which shows data that the break occurs around year 1975. The economical explanation for this break may related to the OPEC energy price rising in 1975 when the oil price raised 10\%, which brought a huge shock to the economic field, including the job market. Therefore there are two different states of the unemployment rates: before and after the oil price change. Moreover, the sum of residual squares for LSTAR model is 9.53, which is the lowest of the three models.

\section{Size property under GARCH $(1,1)$ error}

We turn here to the question of how the size property will be affected when GARCH errors appear. The GARCH $(1,1)$ is the most frequently used due to its simplicity and robustness. Thus we only concentrate the test property when the error of the DGP exhibits $\operatorname{GARCH}(1,1)$. The unit root process with $\operatorname{GARCH}(1,1)$ error is as follows:

$$
\begin{aligned}
& y_{t}=y_{t-1}+u_{t}, \\
& u_{t}=\eta_{t} \sqrt{h_{t}}, h_{t}=w+\alpha u_{t-1}^{2}+\beta h_{t-1} ; \eta_{t} \sim \text { i.i.d. }(0,1), w=1-\alpha-\beta .
\end{aligned}
$$

For the unit root based on the LSE in the linear case, Cook (2006) observed that size distortions of GARCH error are mainly caused by the volatility parameter $\alpha$. Hence, our experiment design will include both the cases where $\alpha \geq \beta$ and $\alpha<\beta$ in the following situations: medium and high GARCH effect: $\alpha+\beta=0.75 ; \alpha+\beta=0.95$. To judge the reasonability of the size property at 5\% nominal size, an unbiased estimated should lay between the approximate $95 \%$ confidence intervals of the actual size $5 \%$. With replication number equal to 10000 , the approximate $95 \%$ confidence interval for the estimated size is: $0.05 \pm 1.96 \sqrt{\frac{0.05(1-0.05)}{10000}}=(0.0457,0.0543)$. The size table of the test under the medium GARCH error is now: 
Table 3. Size property for the test under $\operatorname{GARCH}(1,1)$ error, $\alpha+\beta=0.75$

\begin{tabular}{|llllll|}
\hline & $\alpha=0.3$ & $\alpha=0.35$ & $\alpha=0.4$ & $\alpha=0.45$ & $\alpha=0.5$ \\
$\mathrm{~T}$ & $\beta=0.45$ & $\beta=0.4$ & $\beta=0.35$ & $\beta=0.3$ & $\beta=0.25$ \\
\hline 50 & 0.0897 & 0.0951 & 0.1074 & 0.1088 & 0.1102 \\
\hline 100 & 0.0849 & 0.0891 & 0.0962 & 0.1038 & 0.1032 \\
\hline 250 & 0.0734 & 0.0746 & 0.088 & 0.0935 & 0.0994 \\
\hline 500 & 0.0752 & 0.0769 & 0.0756 & 0.0853 & 0.0885 \\
\hline
\end{tabular}

Table 3 shows that when $\alpha+\beta=0.75$, there exists obvious size distortion which is more serious as $\alpha$ increases and less serious in larger samples. This can be interpreted as follows: when GARCH effect is not high, the size distortion is mainly due to the volatility parameter $\alpha$ and it will be milder when the sample size grows. For the situation of high GARCH effect where $\alpha+\beta=0.95$, the size is over biased as well and we will present it later together with the wavelet improved size in Table 6.

\section{Wavelet improvement of size distortion under GARCH error}

In this section we use the wavelet method to solve the problem of over-rejection under GARCH error. The process is simple: first we generate a new table of critical values where the DGP is the first level boundary wavelet scale coefficients get by Maximal Overlap Discrete Wavelet Transform (MODWT). Next, we apply the test using these scale coefficients instead of the original series. The logic behind this method is that, after wavelet decomposition, the wavelet's high frequency coefficients which contain short time volatility information brought by GARCH $(1,1)$ error are counted off. Those scale coefficients contain all the non stationary information when the original time series follows a unit root process, while the scale coefficients are still stationary when the original time series is stationary. Thus when conducting the unit root test, we use the scale coefficients $V_{t}=\sum_{l=0}^{L-1} g_{l} y_{t-l \bmod T}$ instead of the original data, while $g_{l}$ is 
the scaling filter satisfying: $\sum_{l} g_{l}=1, \sum_{l} g_{l}^{2}=1 / 2, \sum_{l} g_{l} g_{l+2 *_{n}}=0$

Assumption 2: $\left\{w_{t}\right\}$ is a linear process which can be defined as follows: $w_{t}=\psi(L) u_{t}=\sum_{j=0}^{\infty} \psi_{j} u_{t-j}, \psi(1) \neq 0$, and $\sum_{j=0}^{\infty} j\left|\psi_{j}\right|<\infty$.

Theorem 2: Under Assumptions 1 and 2, the asymptotical distributions of the Nonlinear Dickey-Fuller F test statistics will not be influenced when we use wavelet scale coefficients $V_{t}=\sum_{l=0}^{L-1} g_{l} y_{t-l \bmod T}$ instead of original series $y_{t}$ with $y_{t}=y_{t-1}+u_{t}$, where $u_{t}$ fulfills Assumption 1.

For small sample, Monte Carlo experiment gives following critical value table:

Table 4. Critical values for the wavelet improved Nonlinear D-F $F$ test

\begin{tabular}{|lllllllll|}
\hline $\mathrm{T}$ & $99 \%$ & $97.5 \%$ & $95 \%$ & $90 \%$ & $10 \%$ & $5 \%$ & $2.5 \%$ & $1 \%$ \\
\hline 50 & 0.1699 & 0.2600 & 0.3797 & 0.5626 & 3.9150 & 4.7469 & 5.5354 & 6.6924 \\
\hline 100 & 0.2607 & 0.4087 & 0.5792 & 0.8282 & 4.5163 & 5.3862 & 6.2805 & 7.2699 \\
\hline 250 & 0.6007 & 0.8122 & 1.0262 & 1.3412 & 5.4247 & 6.3390 & 7.2056 & 8.3035 \\
\hline 500 & 0.9216 & 1.1318 & 1.3484 & 1.6354 & 5.8999 & 6.8178 & 7.7707 & 8.9749 \\
\hline
\end{tabular}

We can see that as the sample size increase, the critical values will approach the one we obtain from Table 1, which also implies that the distribution will not be influenced asymptotically. The size of the test using the wavelet scale coefficient is still unbiased under $u \sim$ n.i.d. $(0,1)$.We are more interested to see the size property of the wavelet improved test when the original DGP suffered from $\operatorname{GARCH}(1,1)$ error, when $\alpha+\beta=0.75$ and 0.95 , see Table 5 below.

When comparing Table 5 to Table 3, where no wavelet method is applied, we see that although there are only a few unbiased size in Table 5, the over-rejection problem get improved in each grid. 
Table 5. Size property for the test in wavelet under $\operatorname{GARCH}(1,1), \alpha+\beta=0.75$

\begin{tabular}{|llllll|}
\hline & $\alpha=0.3$ & $\alpha=0.35$ & $\alpha=0.4$ & $\alpha=0.45$ & $\alpha=0.5$ \\
$\mathrm{~T}$ & $\beta=0.45$ & $\beta=0.4$ & $\beta=0.35$ & $\beta=0.3$ & $\beta=0.25$ \\
\hline 50 & 0.0597 & 0.0625 & 0.0681 & 0.0628 & 0.0670 \\
\hline 100 & 0.0710 & 0.0638 & 0.0691 & 0.0726 & 0.0700 \\
\hline 250 & 0.0644 & 0.0690 & 0.0711 & 0.0720 & 0.0700 \\
\hline 500 & 0.0669 & 0.0691 & 0.0674 & 0.0740 & 0.0737 \\
\hline
\end{tabular}

For the case when, $\alpha+\beta=0.95$, the wavelet improved size property and the original size which is in () are as follows:

Table 6. Size property for the test under $\operatorname{GARCH}(1,1)$ error, $\alpha+\beta=0.95$

\begin{tabular}{|clllll|}
\hline & $\alpha=0.4$ & $\alpha=0.45$ & $\alpha=0.5$ & $\alpha=0.55$ & $\alpha=0.6$ \\
$\mathrm{~T}$ & $\beta=0.55$ & $\beta=0.5$ & $\beta=0.45$ & $\beta=0.4$ & $\beta=0.35$ \\
\hline 50 & $0.0500(0.0762)$ & $0.0521(0.0745)$ & $0.0514(0.0831)$ & $0.0487(0.0785)$ & $0.0472(0.0754)$ \\
\hline 100 & $0.0609(0.0905)$ & $0.0608(0.0891)$ & $0.0543(0.0875)$ & $0.0543(0.0879)$ & $0.0513(0.0853)$ \\
\hline 250 & $0.0741(0.1013)$ & $0.0732(0.0962)$ & $0.0699(0.0909)$ & $0.0580(0.0901)$ & $0.0629(0.0901)$ \\
\hline 500 & $0.0799(0.1055)$ & $0.0795(0.1116)$ & $0.0718(0.1064)$ & $0.0681(0.0950)$ & $0.0626(0.0908)$ \\
\hline
\end{tabular}

Table 6 shows over-rejection problem of the size is obviously improved, especially when the sample size is small, and where the wavelet improved size is almost unbiased and lies between the $95 \%$ confidence interval. However, when the sample size increases, there is still some size distortion, which may due to the aggregate influence of the GARCH effect in large sample sizes for the near integrated GARCH error when $\alpha+\beta=0.95$.

\section{Conclusions}

In this paper we first propose a nonlinear Dickey-Fuller $F$ test against LSTAR (1) model with time as the transition variable. The asymptotic distribution of the Nonlinear Dickey-Fuller $F$ test statistic is derived while distributions of finite samples 
are obtained by Monte Carlo simulations. The size of the test statistics is unbiased and the power shows good property in larger samples. We also use an empirical example to compare the nonlinear Dickey-Fuller $F$ with traditional Dickey-Fuller $F$ test. Technically speaking, the Nonlinear Dickey-Fuller $F$ test is not very innovative as it is mainly an addition of the nonlinear Dickey-Fuller $\rho$ and $t$ test proposed by $\mathrm{He}$ and Sandberg (2006). The main point of this paper is to show that our test suffered from serious size distortion under medium and high GARCH $(1,1)$ error. To resolve the problem, we use wavelet method as the wavelet scale coefficient can maintain the unit root information while count the GARCH effort off. We show that by using the wavelet method, the asymptotic distribution is not influenced, while the over-rejection problem in small sample size is improved.

\section{References}

Charles A. and Darné O. (2008). “A Note on Unit Root Tests and GARCH Errors: A Simulation Experiment," Communications in Statistics - Simulation and Computation, Vol. 37, Issue 2, pp. $314-319$.

Cook S. (2006). "The Robustness of Modified Unit Root Tests in the Presence of GARCH," Quantitative Finance, Vol. 6, issue 4, pp.359-363.

Dickey D.A. and Fuller W.A. (1979). "Distributions of the Estimators for Autoregressive Time Series with a Unit root," Journal of American Statistical Association, Vol. 74, pp.427431.

Eklund B. (2003). “Testing the Unit Root Hypothesis against the Logistic Smooth Transition Autoregressive Model," Working Paper Series in Economics and Finance from Stockholm School of Economics, No. 546.

Engle R.F. (1982). “Autorregressive Conditional Heteroskedasticity with Estimates of United Kingdom Inflation,” Econometrica, Vol. 50, pp.987-1008.

Fan Y.Q. and Gençay R. (2006). "Unit Root and Cointegration Tests with Wavelets," Canadian Econometric Study Group Meeting, McGill University, Montreal, September 29-30.

Gençay R., Selçuk F. and Whitcher, B. (2001b). An Introduction to Wavelets and Other 
Filtering Methods in Finance and Economics. Academic Press, San Diego.

Grossmann A. and Morelet J. (1984). "Decomposition of Hardy Functions into Square Integrable Wavelets of Constant Shap," SIAM Journal on Mathematical Analysis, Vol. 15, pp. 723-736.

Hamilto J.D. (1994). Time series analysis, Princeton University Press, Princeton NJ.

He C. and Strandberg, R. (2006). "Dickey-Fuller Type of Tests against Non-linear Dynamic Models," Oxford Bulletin of Economics \& Statistics, Vol. 68, No. s1, 2006, pp. 835-86.

Kim and Schmidt P. (1993). "Unit Root Tests with Conditional Heteroskedasticity," Journal of Econometrics 59 pp. 287-300.

Li W.K., Ling S.Q. and Michael M. (2002). "Recent Theoretical Results for Time Series Models with GARCH Errors,” Journal of Economic Surveys, Vol. 16 Issue 3, pp. 245-269.

Ling S.Q. and Li, W.K. (1998). "Limiting Distributions of Maximum Likelihood Estimators for Unstable Autoregressive Moving-Average Time Series with General Autoregressive Heteroscedastic Errors," Annals of Statistics, Vol. 26 (1), pp.84-125.

Luukkonen R.,Saikkonen P. and Teräsvirta. T. (1988). “Testing Linearity against Smooth Transition Autoregressive Models," Biometrika, Vol. 75, pp. 491-499.

Mallat S.G..(1989).“A Theory for Multiresolution Signal Decomposition: the Waveletrepresentation," Pattern Analysis and Machine Intelligence, IEEE Transactions. Vol. 11, Issue. 7, pp. 674-693.

Percival D.B. and Walden A.T. (2000). Wavelet Methods for Time Series Analysis. Cambridge Press, Cambridge.

Peters T.A. and Veloce W. (1988). "Robustness of Unit Root Tests in ARMA Models with Generalized ARCH Errors," Unpublished manuscript, Brock University.

Schleicher C. (2002). “An Introduction to Wavelets for Economists,” Working paper 2002-3. Seo B. (1999). "Distribution Theory for Unit Root Tests with Conditional Heteroskedasticity," Journal of Econometrics, Vol. 91, Issue 1, pp. 113-144.

Teräsvirta T. (1994). "Specification, Estimation, and Evaluation of Smooth Transition Autoregressive Models,"Journal of the American Statistical Association, Vol. 89, pp. 208-218.

Tong H. (1990). Nonlinar time series. Oxford Science Publicatons.

Vidakovic B. (1999). Statistical Modeling by Wavelets. New York: John Wiley and Sons. 